\title{
Evaluation of an Outpatient Pharmacist Consult Service at a Large Academic Medical Center
}

\author{
Melanie Berry, PharmD ${ }^{1}$; Amy Gustafson, PharmD, BCACP'; Maya Wai, PharmD, BCPS ; Alex J. Luli, PharmD, BCACP 4
}

${ }^{1}$ Beth Israel Deaconess Medical Center; ${ }^{2}$ Twinsburg Ambulatory Pharmacy; ${ }^{3}$ University of Arkansas for Medical Sciences, College of

Pharmacy; ${ }^{4}$ University of California San Diego, Skaggs School of Pharmacy and Pharmaceutical Sciences

\section{Abstract}

Objective: To evaluate a novel outpatient pharmacist consult service in a large academic medical center.

Setting: Four outpatient pharmacies that are part of a large academic medical center

Methods: An outpatient pharmacist consult order was created and embedded in the electronic medical record (EMR). Medical center providers utilized this consult order when identifying patients in need of specific services provided by outpatient pharmacists. Descriptive data about each individual consult was collected including number completed, type of service, and duration. Rate of accepted pharmacy recommendations and patient cost savings were also evaluated. A survey was administered at the completion of the study period to assess provider and pharmacist satisfaction with the service. Patient demographic information was collected for those who had a documented completed consult.

Results: A total of 193 consults were completed: 137 immunizations, 37 care affordability, 15 education, 3 polypharmacy and 1 OTC recommendation. 89\% of completed consults took pharmacists 20 minutes or less to complete. Of completed care affordability consults $(n=31), 55 \%$ of patients saved between $\$ 100$ - $\$ 500$ per medication fill. Of providers who completed a survey and utilized the service $(n=12), 83.3 \%$ were extremely satisfied and $16.7 \%$ were satisfied with it. The provider acceptance rate of pharmacist's recommendations was $74 \%$.

Conclusion: Implementation of an outpatient pharmacist consult service provided an alternative method for the utilization of pharmacist provided MTM services in outpatient pharmacies at a large academic medical center. The service was well received by both providers and pharmacists.

Keywords: Pharmacists, Referral and Consultation, Electronic Health Records, Humans, Community Pharmacy Services/organization \& administration, Outpatients

\section{BACKGROUND}

The American Pharmacists Association (APhA) defines Medication Therapy Management (MTM) as a distinct service or group of services that optimize therapeutic outcomes for individual patients, which can occur independent of, or in conjunction with, the provision of a medication product. ${ }^{1}$ MTM services are designed to enhance patient understanding, increase patient adherence to medication regimens, detect adverse drug events and identify patterns of medication overuse and underuse. ${ }^{2}$ The effectiveness of pharmacistprovided MTM services has been documented in various settings with positive health outcomes including lowering blood pressure, lowering LDL cholesterol, and increasing medication adherence. ${ }^{3-5}$ As drug therapy experts, pharmacists are well positioned to offer MTM services in a variety of practice settings, including community pharmacies, primary care clinics, Federally Qualified Health Centers (FQHCs), and outpatient pharmacies operating as part of a health system. ${ }^{6}$ Over time, MTM services have evolved from an initial focus on patient education in acute medications and diseases towards long-term management of chronic medications and disease states. ${ }^{7}$

Corresponding author: Alex J. Luli, PharmD, BCACP

Health Sciences Assistant Clinical Professor

University of California San Diego

Skaggs School of Pharmacy and Pharmaceutical Sciences 9500 Gilman Drive, Mail Code 0657. La Jolla, CA 92093-0657

Phone: 858.534.5750; Email: aluli@health.ucsd.edu
Many pharmacies, especially those in the community, that provide MTM services utilize a similar workflow to identify eligible patients, document interventions, and communicate with other healthcare providers. This workflow typically involves, and is heavily dependent on, the use of third-party platforms to help pharmacies complete a majority of the necessary workflow steps. For example, third-party platforms identify patients that are eligible for MTM services (based on their insurance plan), provide a user-friendly interface to complete documentation and billing, and provide standardized forms to aid in communicating with healthcare providers. ${ }^{7,8}$ Despite their routine use and positive characteristics, many challenges with these platforms remain. A patient's health information may be inaccurate, incomplete, and include partial prescription fill histories. The platforms also lack the capability to directly communicate with other systems used by providers for patient care, making it difficult for a pharmacist to relay documented interventions. ${ }^{9}$ In addition, patients that are identified as eligible for services may not visit the pharmacy routinely, which may create inconsistency and confusion for both the patient and the pharmacist. Finally, many third-party platforms suggest potential drug therapy problems. Although helpful in some cases, many are based on limited patient specific health information. Therefore, efforts to resolve these issues are unproductive. For example, a third-party platform may identify a patient who has diabetes without a prescription fill history of a statin. However, this patient may be intolerant to statins and therefore efforts to recommend a statin are clinically inappropriate. 
Despite these challenges, little evidence exists suggesting the best approach to address them. One study by Dolor and colleagues discussed lessons learned during implementation of a pharmacist-provided MTM service in a primary care setting. They discovered off-site implementation of MTM services resulted in decreased utilization by both patients and providers. The authors concluded that integrating MTM services during existing clinic appointments may improve participation rates. ${ }^{10}$

In a survey of physicians regarding preferences for communication of MTM services provided in community pharmacies, Guthrie and colleagues found the greatest communication barriers to be lack of time and inefficient communication practices. $^{11}$ Pharmacists practicing in outpatient pharmacies that are part of a health system have the unique opportunity to offer MTM services that overcome the barriers described above. These pharmacies can utilize access to a patient's medical record and accessibility to other members of the healthcare team to enhance provision of MTM services.

Objectives: To describe the implementation and evaluation of a pilot outpatient pharmacist consult service. Data was collected on type and duration of completed consult services, provider acceptance rate of pharmacist's recommendations, and patient's cost savings. Provider and pharmacist satisfaction with the service was also assessed.

Setting: Cleveland Clinic Health System (CCHS) is comprised of a 1,400-bed tertiary, academic medical center, 17 regional hospitals (including five in Southeast Florida), 45 community health facilities called Family Health Centers (FHCs), and numerous community physician practices. FHCs offer comprehensive services including lab, imaging, physical and occupational therapy, primary and specialty care, same day surgery, emergency medicine, and pharmacy services. Outpatient prescription services are provided by 20 outpatient, ambulatory pharmacies, seven of which are embedded in FHCs. This pilot study was conducted at four of these pharmacies.

\section{Practice Description}

Cleveland Clinic outpatient pharmacies dispense prescriptions for patients seen in the FHC, ambulatory surgery center (ASC), emergency department, and for CCHS employees and their dependents. Additional clinical services are provided including comprehensive medication reviews, durable medical equipment, investigational drug use, immunizations, payerinitiated MTM, and adherence counseling. Pharmacists have access to the electronic medical record (EMR) to review patient specific information, document clinical services, and communicate with other members of the healthcare team. Pharmacists support providers by answering drug information questions, recommending alternate therapies, identifying and resolving drug-related problems, and assist with computerized physician order entry (CPOE). Previously, there was no formal process for providers to consult outpatient pharmacists and most consultation was conducted on-the-fly via telephone with agents of the provider. [Note: CCHS has ambulatory care pharmacists embedded in a variety of settings across the institution conducting comprehensive medication management. The service described here only applies to those pharmacists who are primarily practicing in the outpatient, ambulatory pharmacies.]

\section{Practice Innovation}

An outpatient pharmacist consult order was developed within the EMR to capture provider-identified MTM opportunities, streamline patient handoff, and provide transparent documentation of pharmacist services for improved communication between the healthcare team. This consult order was designed for use by internal and family medicine physicians and other providers in the health system. Consults were organized into five categories: care affordability, immunization, medication education, polypharmacy, and overthe-counter (OTC) recommendation. Each consult category was designed to address a specific patient need and were further classified into sub-types, which are listed in Table 1. Four outpatient pharmacies were selected as participating pilot sites. These pharmacies were primarily selected based on their location (within FHCs). Each pharmacist manager at participating pharmacies was responsible for communicating the details of this service to all internal and family medicine providers at their location. This was accomplished by a combination of individual one-on-one meetings, presenting at standing staff meetings, email notifications, and inclusion in system-wide newsletters.

After identifying a patient for referral, providers entered the consult order electronically via the EMR and selected one of the four participating pilot site pharmacies. To enhance service integration, provider acceptance, and ease of use, the consult order was designed in the same style and workflow as preexisting consult orders. The consult order informed pharmacists of the reason for patient referral, preferred pharmacy location (based on patient and/or provider preference) and any additional information provided by the referring provider, such as related diagnosis. Within 48 hours of receiving notification, pharmacists made three attempts to contact patients to schedule in-person (preferred) or telephone appointments to complete the consult. If a patient could not be reached after 3 attempts, the referring provider was notified and the consult closed. Following completion of the consult, pharmacists documented the service and relevant notes in the patient's EMR. A copy was forwarded to the referring provider, if requested. Standardized documentation templates were created in the EMR to ensure consistent documentation and data review. When pharmacists identified drug-related problems that required prescriber intervention, such as modifying medication therapy, high priority notes were sent to the referring provider via the EMR for follow up. This is consistent with practice across the institution. 


\section{Evaluation}

Data from the consult service was collected during the pilot study period - September 21, 2017 through January 31, 2018. The following data was collected for all completed consults: time spent, pharmacy location, number of recommendations made and accepted by providers, and patient demographics. For the care affordability consult, estimated patient cost savings per fill was collected. This calculation assumed a patient was adherent to the prescribed therapy and would utilize their medication as directed. Cost savings were then calculated based on the reduction in out-of-pocket costs that were achieved due to interventions by the pharmacist. The methods employed to save patients money included recommending alternate therapies, contacting insurance plans to determine preferred formulary alternatives, and utilization of manufacturer copay saving programs. Incomplete consult notes were excluded, as well as any MTM services that were provided without a provider consult order (pharmacists were still able to conduct routine MTM services for any patient, independent of a consult order, as deemed appropriate by that pharmacist). To assess satisfaction with the consult service, a survey was distributed to pharmacists and providers at the end of the pilot period.

\section{RESULTS}

In total, 198 consult orders were sent to the pilot pharmacies from 23 providers. Of those, 193 consults were successfully completed by pharmacists (97\%). One patient refused the service and four were unable to be reached. Patient demographics are summarized in Table 2 . All five consult types were ordered: Immunizations (70.9\%, n=137), care affordability (19.2\%, $n=37)$, medication education (7.8\%, $n=15)$, polypharmacy $(1.6 \%, n=3)$ and OTC recommendation $(0.5 \%$, $\mathrm{n}=1)$. The most common immunization requested was live zoster vaccine $(n=77)$, followed by diphtheria/tetanus toxoids; pertussis vaccine $(n=54)$, pneumococcal 13 -valent conjugate vaccine $(n=5)$ and pneumococcal vaccine polyvalent $(n=1)$. Number of consult types completed per month is shown in Figure 1. Of the completed care affordability consults ( $n=31), 26$ $(84 \%)$ patients saved up to $\$ 500$ per fill of medication and 5 (16\%) patients saved > \$501 (Figure 2). Time spent completing each encounter was documented for 189 consults: 169 (89\%) were completed in 20 minutes or less (Figure 3).

At the end of the pilot study period, 15 providers completed the satisfaction survey. Of the providers who utilized the service $(n=12), 10(83.3 \%)$ and $2(16.7 \%)$ were either extremely satisfied or satisfied with the service, respectively. 11 (73.3\%) providers reported they were highly likely to use the service in the future, while $2(13.3 \%)$ reported likely to use and 2 (13.3\%) were willing to use the service, but have reservations. Providers accepted 28 recommendations made by pharmacists (74\%). Of the pharmacists who completed the survey $(n=6), 2(33.3 \%)$ and $4(66.7 \%)$ were extremely satisfied or satisfied with the service, respectively. $50 \%$ of pharmacists responded they were highly likely and $50 \%$ were likely to use the service in the future.

\section{DISCUSSION}

During the pilot study period, pharmacists provided a high number of vaccinations, completed several types of patient education, comprehensively reviewed medication lists and saved patients money on their prescription copays. Survey results indicated providers and pharmacists were satisfied while utilizing this service, and most were likely to use in the future. Patients who were referred for these services tended to be older (median age $=66.9$ years) and on multiple medications (median $=10$ ). These are common patient characteristics for those who utilize MTM services in general, since many eligible patients are Medicare beneficiaries and take multiple medications. ${ }^{12}$ of note, since patients in our study could have more than one consult type (e.g., a provider recommends an immunization and medication education), demographics reflect 185 unique patients, not the total consult orders placed. Based on past experience providing MTM services in outpatient pharmacies, we observed the process leading up to providing a useful service can be inefficient and heavily dependent on third-party platforms. In many cases, significant time is invested to research and contact patients who do not need or refuse MTM services. Payer-based MTM referrals are often formulary driven rather than clinically significant, patient-specific interventions. The outpatient pharmacy consult service described here shifts the identification of patients to the provider. Although pharmacists are still able to provide MTM services to patients as appropriate, our service streamlined this process since the provider routed pharmacy consults through the EMR in the same manner to placing other consults. This allowed for closed-loop communication directly between the provider and the pharmacy, and documentation of the completed consult in the shared EMR. This aligned with standard communication practices within the health system for patient-care related information. Additionally, coordinating and documenting all patient-care activities within the same EMR is a benefit beyond current capabilities of most standalone retail pharmacies and traditional payer-identified MTM services. The high service completion rate (97\%) helps support the theory that a collaborative approach to identifying patients eligible for MTM services may be effective. We feel this contributed to the satisfaction and acceptance rate from both providers and pharmacists with the service.

There were several limitations during service implementation and completion. Pharmacists sometimes utilized the documentation templates without a receiving consult order from a provider. For example, many immunizations completed did not have a consult order placed first, and patients may have been verbally instructed by a provider to get an immunization in the pharmacy. These patients were still given an immunization, if appropriate. Additional training was provided to pharmacists and prescribers to ensure consults were appropriately ordered and documented. Despite the study period (peak months for influenza immunization), influenza vaccinations were offered and administered through the provider's office or community walk-in clinics, not outpatient 
pharmacies, and thus were not included in the consult order. If offered, as is done in many pharmacy settings, vaccination consult orders would have likely been higher. Also, due to the anticipated release of recombinant herpes zoster vaccine (Shingrix ${ }^{\circledR}$ ) during the study period, the number of live zoster vaccines administered was likely impacted.

Our purpose for selecting the specified consult order categories (immunization, care affordability, medication education, polypharmacy, and OTC recommendation) was to replicate those services that are readily available (and commonly provided) in the outpatient pharmacy setting (including at stand-alone community pharmacies). By streamlining the process for the provider, our aim was to "catch" those patients who may benefit from MTM services as they are identified by a provider during the course of routine care. Instead of a provider simply telling a patient they should talk to their pharmacist about affording their medications, an action can be taken (ie ordering an outpatient pharmacist consult), helping to ensure a patient care service is completed and documented.

In addition, another limitation is the lack of a pharmacoeconomic analysis relating to this service. As mentioned, the intent of this pilot consult service was to describe an alternative way to incorporate MTM services into the typical workflow of a community pharmacy. Further, no direct billing of the consult service to insurance payers or patients took place. The service itself was provided free of charge for patients. For consult services that resulted in a product or service with billing procedures already established (e.g., administration of a vaccine or new medication prescription), billing followed the standard operating procedures of the pharmacy. Future directions could involve billing of pharmacist provided services, especially for those consult types that are also covered under certain health plans. For example, a patient referred to the pharmacist for a comprehensive medication review who is also a Medicare Part D plan beneficiary. In such a case, the pharmacy could bill the patient's insurance plan directly for providing the comprehensive medication review. Outside of this example, however, reimbursement for pharmacist provided services remains a major barrier for implementation of new and expansion of existing services. Cash payment for pharmacist provided services could also be considered, especially for consults such as care affordability - where patient savings on medications could help justify the cost of the service. As payment models continue to evolve, a consult-based program for MTM services may be advantageous, since all documentation is entered in the EMR and could follow similar billing practices for other healthcare services a patient might receive.

Generalizability to community pharmacies without access to patient's EMRs may be low due to limited patient data and lack of an integrated interface with other providers. In addition, interprofessional relationships have been identified as a barrier to implementing MTM services in the outpatient setting ${ }^{11}$. Future studies should evaluate characteristics of providers utilizing the service, in addition to clinically relevant patient characteristics (e.g., diabetes control based on Hemoglobin A1c values) and patient satisfaction. This may help identify aspects of those likely to use this service and/or unrecognized barriers to widespread adoption of similarly designed MTM services. Further, collaboration between pharmacies and nearby providers should be explored for sharing EMR access to patient information. For example, this type of service could be established as part of physician-pharmacist collaborative practice agreements. Granting external community pharmacies access to EMR software would likely improve the quality of patient care and communication between pharmacists and practitioners across multiple practice settings.

There are opportunities to increase the number and types of pharmacist-provided services that can be ordered via consult. Based on results of this pilot study, the pharmacy consult service was implemented within all Cleveland Clinic outpatient ambulatory pharmacy locations in $1^{\text {st }}$ quarter of 2018. Consult services were also expanded to include medication synchronization and medication adherence, with optional referral to adherence packaging services. To maintain the service, continued education and advertisement to providers and pharmacists may be necessary. Routine quality assurance reviews are recommended to ensure adherence to protocol, quality of patient care, and caliber of pharmacist communication.

\section{CONCLUSION}

Implementation of an outpatient pharmacy consult service enabled pharmacists to conduct MTM services in an alternative, streamlined process. Physicians, pharmacists, and other providers indicated satisfaction with the service and were likely to continue use.

\section{Previous Presentations: \\ Vizient University at Midyear (Orlando, FL), Poster \\ Presentation, December 2017 \\ Great Lakes Pharmacy Residency Conference (Lafayette, IN), Podium Presentation, April 2018 \\ Ohio College of Clinical Pharmacy Spring Meeting (Rootstown, $\mathrm{OH})$, Podium Presentation, June 2018}

Acknowledgements: Thank you to all of the pharmacists, technicians, and staff that supported operations and patient care throughout implementation and expansion of this project. 
Conflict of Interest: We declare no conflicts of interest or financial interests that the authors or members of their immediate families have in any product or service discussed in the manuscript, including grants (pending or received), employment, gifts, stock holdings or options, honoraria, consultancies, expert testimony, patents and royalties.

Funding: This research did not receive any outside funding

IRB: This article describes a retrospective review of a quality improvement project. It was deemed exempt by the study location Institutional Review Board as a quality assessment/quality improvement study.

\section{References}

1. Bluml BM. Definition of Medication Therapy Management: Development of Professionwide Consensus. J Am Pharm Assoc. 2005;45(5):566-572. doi:10.1331/1544345055001274

2. Pellegrino AN, Martin MT, Tilton JJ, Touchette DR. Medication therapy management services: definitions and outcomes. Drugs. 2009;69(4):393-406. doi:10.2165/00003495-200969040-00001

3. Theising KM, Fritschle TL, Scholfield AM, Hicks EL, Schymik ML. Implementation and Clinical Outcomes of an Employer-Sponsored, Pharmacist-Provided Medication Therapy Management Program. Pharmacotherapy. 2015;35(11):e159-163. doi:10.1002/phar.1650

4. Tsuyuki RT, Johnson JA, Teo KK, et al. A randomized trial of the effect of community pharmacist intervention on cholesterol risk management: the Study of Cardiovascular Risk Intervention by Pharmacists (SCRIP). Arch Intern Med. 2002;162(10):1149-1155.
5. Ryan R, Santesso N, Lowe D, et al. Interventions to improve safe and effective medicines use by consumers: an overview of systematic reviews. Cochrane Database Syst Rev. 2014;(4):CD007768. doi:10.1002/14651858.CD007768.pub3

6. CDC. Using Medication Therapy Management to Treat Chronic Disease. Centers for Disease Control and Prevention. Published May 30, 2019. Accessed August 13, 2019.

https://www.cdc.gov/dhdsp/pubs/guides/bestpractices/pharmacist-mtm.htm

7. Barnett MJ, Frank J, Wehring $\mathrm{H}$, et al. Analysis of pharmacist-provided medication therapy management (MTM) services in community pharmacies over 7 years. J Manag Care Pharm JMCP. 2009;15(1):18-31. doi:10.18553/jmcp.2009.15.1.18

8. Brewer J, Kumbera P. Establishing a Web-based program for reimbursement for medication therapy management services. Am J Health Syst Pharm. 2006;63(19):1806-1809. doi:10.2146/ajhp060320

9. Lounsbery JL, Green CG, Bennett MS, Pedersen CA. Evaluation of pharmacists' barriers to the implementation of medication therapy management services. J Am Pharm Assoc. 2009;49(1):51-58. doi:10.1331/JAPhA.2009.07158

10. Dolor RJ, Masica AL, Touchette DR, Smith SR, Schumock GT. Patient safety-focused medication therapy management: challenges affecting future implementation. Am J Manag Care. 2012;18(7):e238244.

11. Guthrie KD, Stoner SC, Hartwig DM, et al. Physicians' Preferences for Communication of PharmacistProvided Medication Therapy Management in Community Pharmacy. J Pharm Pract. 2017;30(1):1724. doi:10.1177/0897190015585764

12. Viswanathan M, Kahwati LC, Golin CE, et al. Executive Summary. Agency for Healthcare Research and Quality (US); 2014. Accessed August 27, 2019. https://www.ncbi.nlm.nih.gov/books/NBK294482/ 
Table 1. Consult Category Definitions and Sub-types

\begin{tabular}{|c|c|}
\hline Category type & Sub-types \\
\hline Immunization & $\begin{array}{ll}\text { - } & \text { Zoster vaccine live } \\
\text { - } & \text { Pneumococcal 13-valent conjugate vaccine } \\
\text { - } & \text { Pneumococcal vaccine polyvalent vaccine } \\
\text { - } & \text { Tetanus, diphtheria, pertussis vaccine } \\
\end{array}$ \\
\hline Care affordability & $\begin{array}{l}\text { - Uninsured patient } \\
\text { - } \quad \text { Non-formulary/non-preferred medication therapy } \\
\text { - } \quad \text { Annual deductible applied to prescription copayment } \\
\text { - } \quad \text { Medicare coverage gap* }\end{array}$ \\
\hline Medication education & $\begin{array}{ll}\text { - } & \text { Device teaching (e.g., glucometer) } \\
\text { - } & \text { Injection technique (e.g., insulin) } \\
\text { - } & \text { Administration technique (e.g., inhaler) } \\
\text { - } & \text { General medication information } \\
\text { - } & \text { Drug interaction resolution } \\
\text { - } & \text { Adverse drug event }\end{array}$ \\
\hline Polypharmacy & $\begin{array}{ll}\text { - } & \text { General drug information } \\
\text { - } & \text { Comprehensive medication review } \\
\text { - } & \text { Medication adherence support } \\
\text { - } & \text { Schedule daily medication doses } \\
\end{array}$ \\
\hline $\begin{array}{l}\text { Over-the-counter (OTC) } \\
\text { recommendation }\end{array}$ & $\begin{array}{ll}\text { - } & \text { Pain/fever } \\
\text { - } & \text { Heartburn } \\
\text { - } & \text { Constipation } \\
\text { - } & \text { Cough/cold } \\
\text { - } & \text { Allergic rhinitis } \\
\text { - } & \text { Vitamin, mineral, and/or herbal supplement } \\
\text { - } & \text { Nicotine replacement therapy }\end{array}$ \\
\hline
\end{tabular}

*based on guidelines set by the Centers for Medicare \& Medicaid Services for study year (2017)

Table 2. Patient Demographics $(n=185)$

\begin{tabular}{|l|c|}
\hline Characteristic & n (\%) \\
\hline Age (in years) & 66.9 \\
\hline Median & 30.1 - 93.1 \\
\hline Range & $110(59.5)$ \\
\hline Sex & $145(78.5)$ \\
\hline Female & $32(17.2)$ \\
\hline Race & $8(4.3)$ \\
\hline White & $17.2+/-13.3$ \\
\hline Black & $11.4+/-6.6$ \\
\hline Other/decline & \\
\hline No. of comorbidities & \\
\hline Mean +/- SD & \\
\hline No. of medications & \\
\hline Mean +/- SD &
\end{tabular}


Figure 1: Number of Consult Types Completed per Month

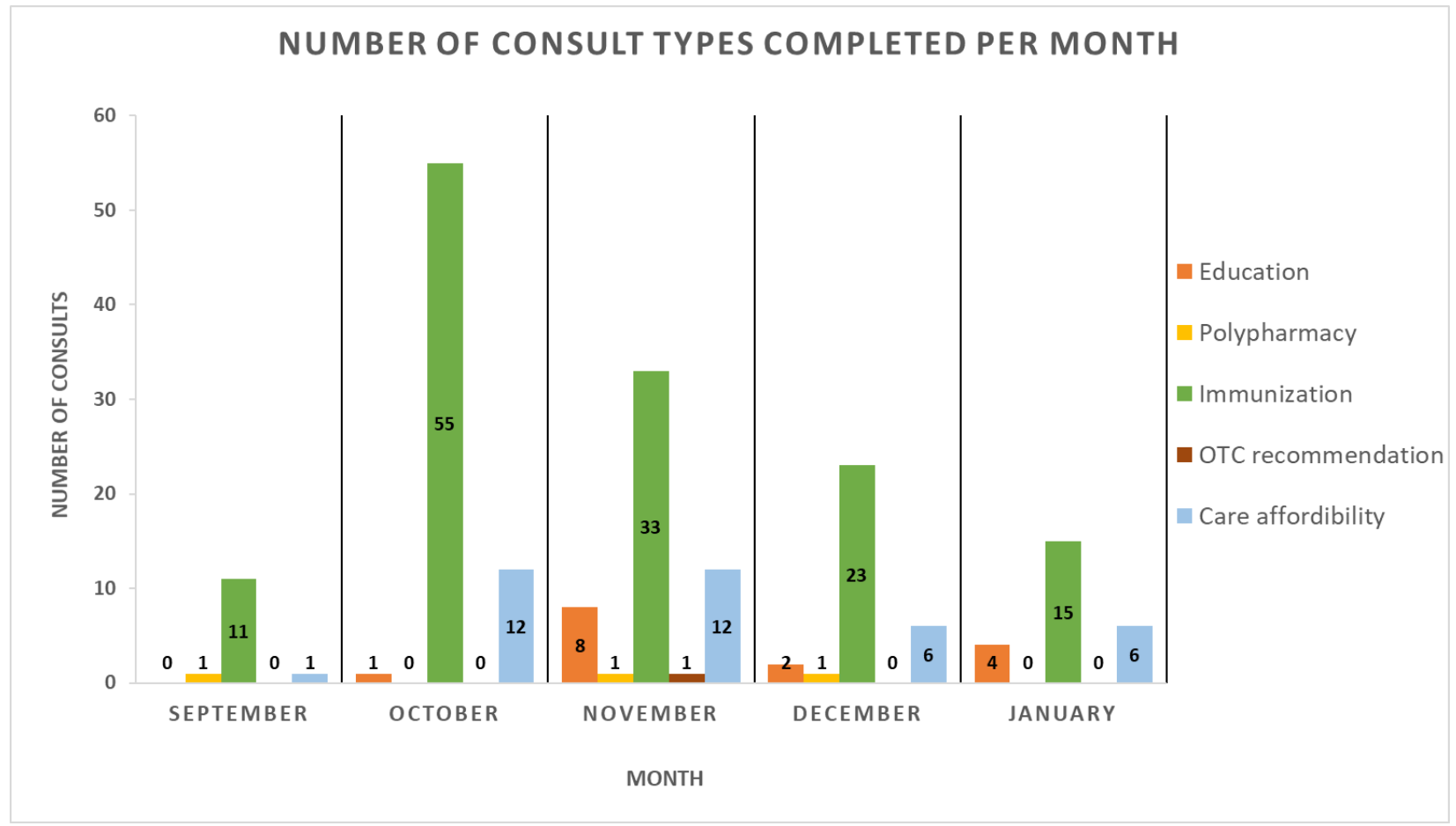

Figure 2: Patient Cost Savings per Medication Fill

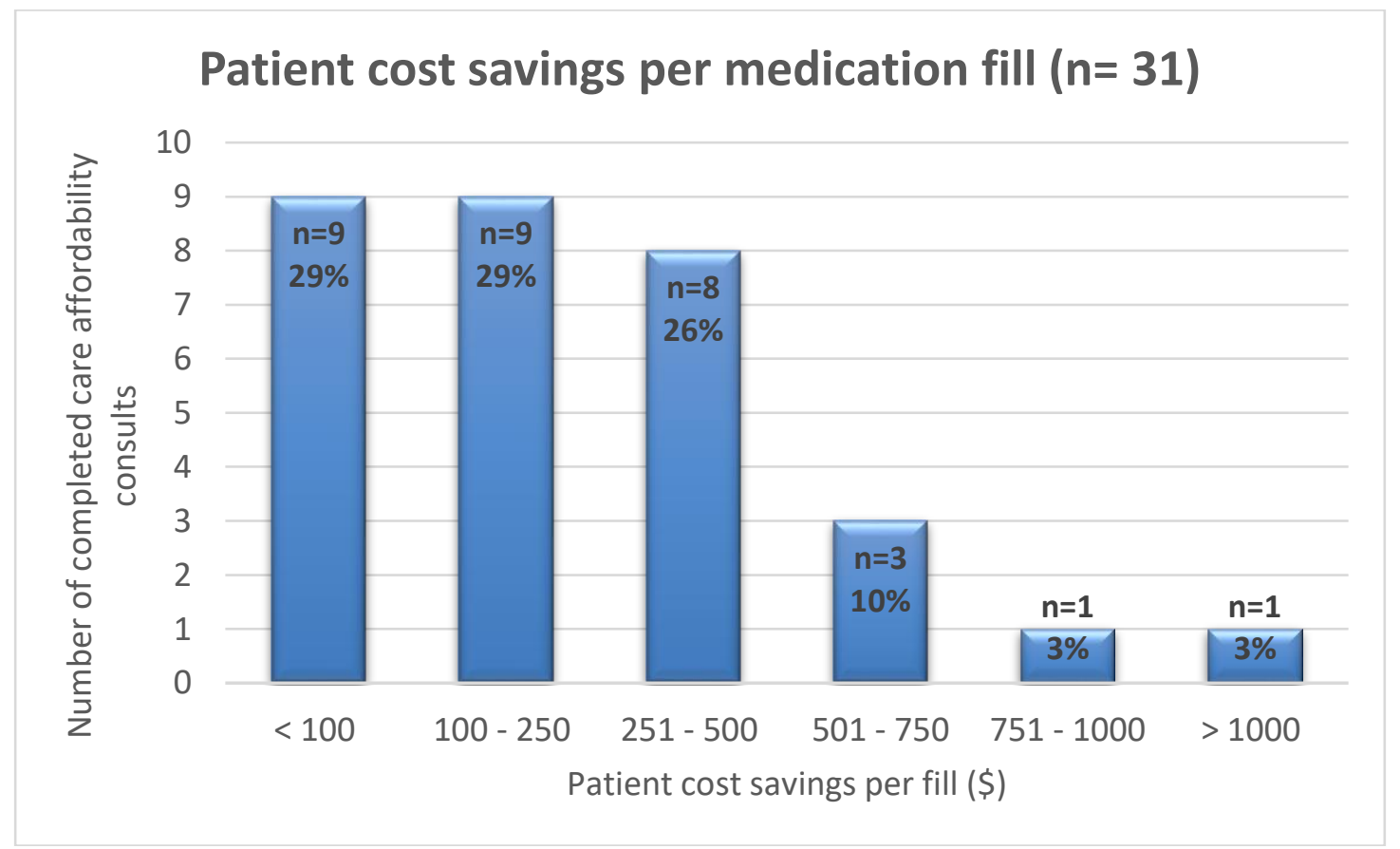


Figure 3: Pharmacist Time Spent on Completing Consults

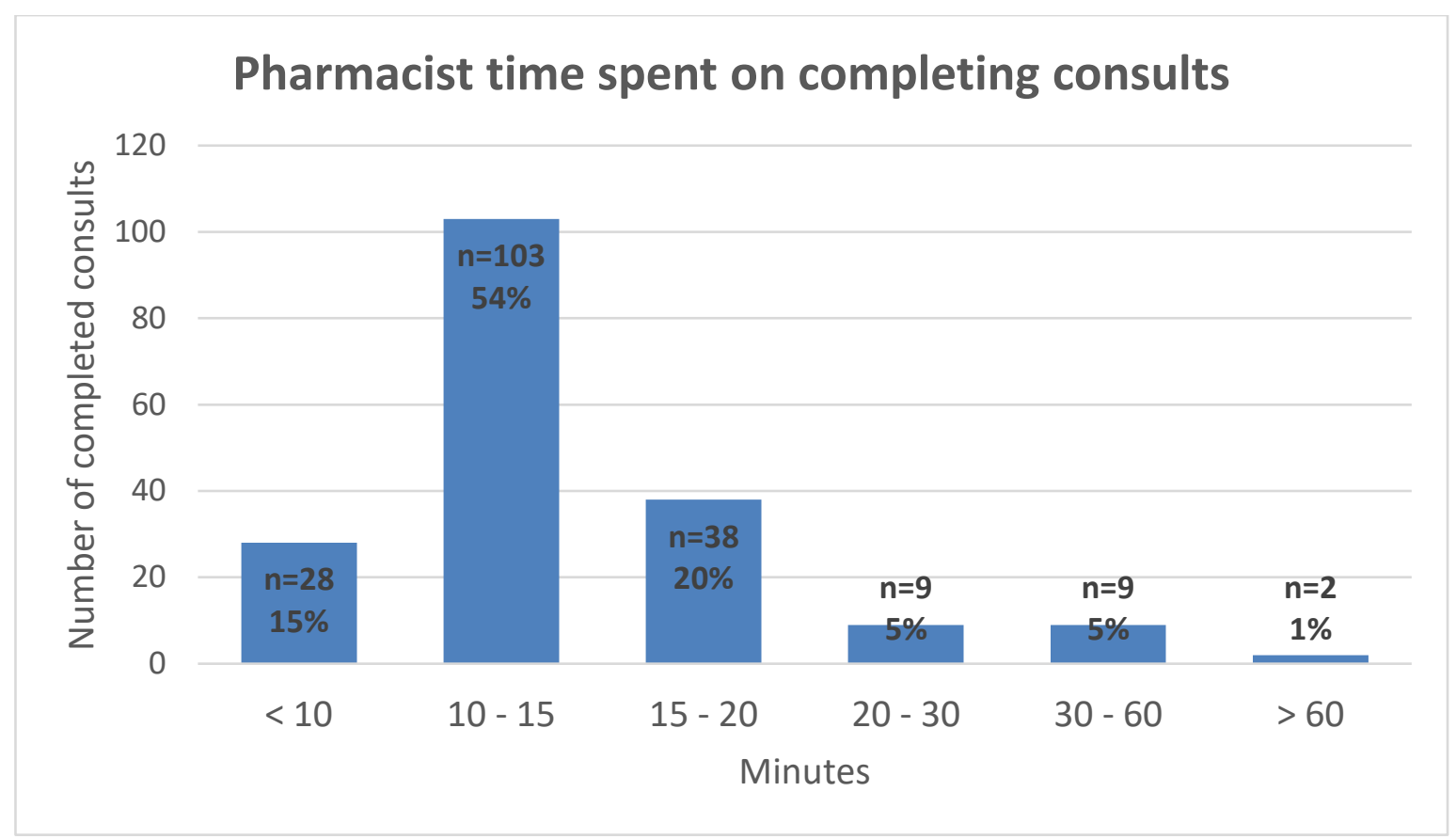

